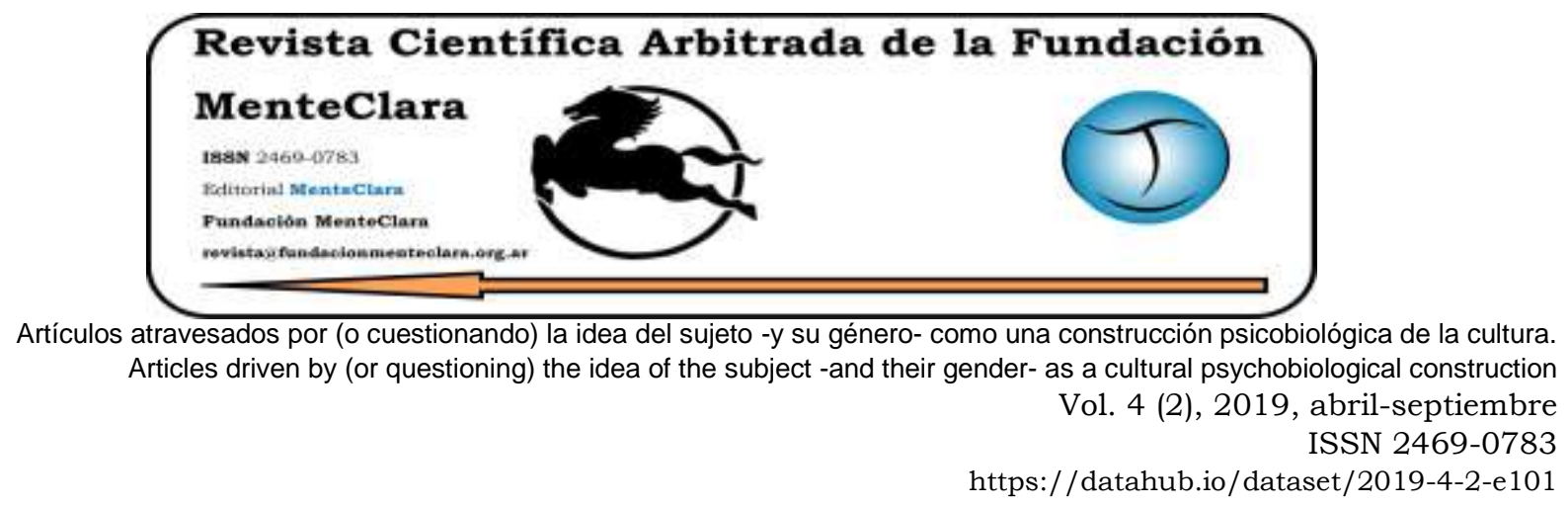

\title{
SOLUCIONES AL SUFRIMIENTO DE LOS OPRIMIDOS: DE LA REVOLUCIÓN CULTURAL A LA EVOLUCIÓN CULTURAL
}

\author{
SOLUTIONS TO THE SUFFERING OF THE OPPRESSED: FROM CULTURAL \\ REVOLUTION TO CULTURAL EVOLUTION
}

Ratan Lal Basu rlbasu@rediffmail.com

Presidency College, Calcutta \& University of Calcutta, India.

Cómo citar este artículo / Citation: Basu, R. L. (2019). "Soluciones al sufrimiento de los oprimidos: De la Revolución Cultural a la Evolución Culturab. Revista Científica Arbitrada de la Fundación MenteClara, 4(2) abril-septiembre 2019, 133-152.

DOI: https://doi.org/10.32351/rca.v4.2.101

Copyright: (C) 2019 RCAFMC. Este artículo de acceso abierto es distribuido bajo los términos de la licencia Creative Commons Attribution 4.0 International License (CC BY 4.0). Recibido: 02/08/2019. Aceptado: 18/09/2019 Publicación online: 30/10/2019

Conflicto de intereses: Ninguno que declarar.

\section{Resumen}

En este artículo consideramos el concepto de la Revolución Cultural desde una perspectiva no convencional y enfatizamos que, en lo que respecta al concepto básico, la Revolución Cultural tiene especial relevancia para la sociedad moderna y puede concebirse como un esfuerzo colectivo para asegurar la elevación de la mente humana y las modalidades psíquicas como un todo. Nos esforzamos por resaltar la correspondencia del concepto de la Revolución Cultural con dos formas casi similares de profundización en las causas de las enfermedades que aquejan a la sociedad humana desde tiempos inmemoriales. La primera comparación se hace con los puntos de vista del filósofo moral y economista Adam Smith, que divide los sentimientos humanos en dos categorías principales, a saber: elementos más nobles y elementos más básicos y atribuye todas las enfermedades de la sociedad humana al último grupo de sentimientos. La segunda posición corresponde a la antigua filosofia india sankhya, que clasifica los modos de vida humanos en tres categorías: tamasika, rajasika y satvika, y mantiene los últimos dos modos responsables de todas 
las enfermedades que afligen la vida humana. Sin embargo, ni la filosofia de Smith ni la sankhya proporcionan ninguna guía para la transición eficaz de la psiquis humana al fin deseado. En este contexto el concepto de Revolución Cultural se vuelve relevante. El concepto, interpretado desde un punto de vista radicalmente diferente de la interpretación maoísta, y, por lo tanto, concebido como un proceso evolutivo en lugar de una revolución destructiva, puede permitirnos diseñar un proceso viable de transición de valores humanos y psíquicos desde los puntos de vista de Smith y la filosofia sankhya.

\section{Abstract}

In this article we look upon the concept of the Cultural Revolution from an unconventional perspective and emphasize that so far as the basic concept is concerned, Cultural Revolution has special relevance for the modern society and it may be conceived as a collective endeavour to ensure uplift of human mind and psychic modes as a whole. Here we endeavour to highlight the correspondence of the concept of the Cultural Revolution to two almost similar ways to go into the depth of the causes of maladies afflicting human society from time immemorial. The first comparison is made with the views of the moral philosopher and economist Adam Smith who divides human sentiments into two major categories, viz. nobler elements and baser elements and attributes all maladies of human society to the later group of sentiments. The second view corresponds to the ancient Indian Sankhya Philosophy, which classifies human modes of living into three categories, viz. tamasika, rajasika and satvika, and holds the latter two modes responsible for all maladies that afflict human living. However, neither Smith nor Sankhya philosophy provide any guidelines for efficacious transition of human psyche to the desired end. In this context, the concept of the Cultural Revolution becomes relevant. The concept, interpreted from a radically different standpoint from Maoist interpretation, and therefore conceived as an evolutionary process rather than a destructive revolution, may enable us to device a viable transitional process of human values and psychic from both the Smithian and the Sankhya standpoints.

Palabras Claves: transición social; evolución cultural; igualdad; fraternidad

Keywords: social transition; cultural evolution; equality; fraternity 


\section{Introducción}

La Revolución Cultural en la República Popular de China -1966-1977ahora es una cuestión de historia y ha sido registrada como una horrible agitación de una década que ha causado estragos en. En retrospectiva, las críticas también se han dirigido contra las premisas teóricas de la Revolución Cultural y muchos críticos han ido tan lejos como para llamarla Fantasía Maoísta.

Sin embargo, este artículo considera el concepto de la Revolución Cultural desde una perspectiva poco convencional y enfatiza que, en lo que respecta al concepto básico, la Revolución Cultural tiene una relevancia especial para la sociedad moderna y puede concebirse como un esfuerzo colectivo para asegurar la elevación de la mente humana y los modos psíquicos en su conjunto.

De hecho, en este artículo, nuestro propósito es relacionar el concepto de la Revolución Cultural con dos formas casi similares de profundizar en las causas de las enfermedades que afligen a la sociedad humana desde tiempos inmemoriales. Ambos, aunque presentados en dos formatos aparentemente diferentes, son esencialmente los mismos, se esfuerzan por destacar la psique humana como la causa de todas las enfermedades que afectan a la sociedad.

Uno de esos puntos de vista ha sido presentado por el filósofo moral y economista Adam Smith, que divide los sentimientos humanos en dos categorias principales, a saber: elementos más nobles y elementos más bajos y atribuye todas las enfermedades de la sociedad al grupo posterior de sentimientos. 
La segunda visión corresponde a la antigua filosofia india de Sankhya ${ }^{1}$ según la cual los modos de vida humanos son de tres categorias, a saber: tamasika, rajasika y satvika. Las enfermedades humanas según los aforismos de Sankhya surgen de los modos de vida tamasika y rajasika.

Para la solución a largo plazo de los problemas básicos de la sociedad sobre la base de la visión smithiana, los sentimientos humanos más básicos deben ser reemplazados por sentimientos más nobles; y según el punto de vista de Sankhya, la eliminación sostenible de enfermedades requiere la transición de los modos tamasika y rajasika al modo satvika.

De hecho, ambos enfoques se reducen al mismo esfuerzo. Los sentimientos básicos de Smith no son más que manifestaciones de los modos tamasika y rajasika. Desafortunadamente, ni la filosofia de Smith ni la de Sankhya han proporcionado pautas eficaces para una transición eficaz de la psique humana requerida por ellos para la elevación de la sociedad.

Aquí viene la relevancia del concepto de la Revolución Cultural. El concepto, interpretado desde un punto de vista radicalmente diferente de la interpretación marxista o maoísta, puede proporcionarnos la transición viable de los valores humanos y psíquicos tanto desde el punto de vista de Smith como del de Sankhya.

Antes de aventurarnos en este esfuerzo hacia la reinterpretación y revalorización del concepto de la Revolución Cultural para resolver los problemas básicos de la sociedad a través de la elevación psíquica de los hombres, veamos primero la turbulenta Revolución Cultural Maoísta, su teoria, sus premisas ideológicas y sus horribles impactos en el marco sociopolítico y económico de la RPC.

\footnotetext{
${ }^{1}$ Hay seis filosofias védicas: La historia de Gautama, Mimamsa de Jaimini, Vaisheshika de Kanada, Sankhya de Kapila y Yoga de Patanjali.
} 


\section{Revolución Cultural de Mao}

Marx no se dio cuenta de que las causas de la pobreza, la desigualdad, la explotación y enfermedades similares no residen en la propiedad privada, las relaciones familiares, el estado o cualquier otro fenómeno visible, sino que se encuentra en lo profundo de la naturaleza humana, en los elementos poco éticos como la codicia, el orgullo y los celos.

Entonces, la erradicación de las enfermedades, si es posible, debe lograrse mediante algún proceso que reduzca la prevalencia de estos vicios básicos en la mente humana. Aparentemente, parece que a través de la Revolución Cultural, Mao queria luchar contra estos vicios básicos de la mente humana que probablemente sean responsables de las enfermedades en el mundo material. Sin embargo, muchos factores hacen dudoso si la Revolución Cultural de Mao apuntó a la erradicación de los vicios primordiales inherentes a la mente humana.

Los siguientes acontecimientos llevaron a Mao a recurrir a la Revolución Cultural:

Aumento de poder en el Partido Comunista del grupo pragmático. Este grupo se opuso fuertemente a los radicales maoístas como Jiang Qing, la esposa de Mao; Lin Biao, el Ministro de Defensa; Chen Boda; Kang Sheng y Wang Donxing.

Los líderes liberales -como Deng Xiaopinging, Liu Shaoqui y el primer ministro Zhou Enlai-, con opiniones radicales maoístas opuestas se habían dado cuenta por la experiencia de China con el socialismo en el transcurso de una década -especialmente los horribles resultados del llamado "Gran salto hacia adelante"-, que los experimentos de Mao serían desastrosos para el desarrollo económico de China y no había otra salida que emprender reformas drásticas y la adopción del camino capitalista o burgués del desarrollo económico. 


\section{El gran salto adelante}

Mao quería adoptar politicas radicales para pasar directamente de una economía semifeudal a una economía socialista en un corto período de tiempo.

El segundo plan quinquenal -1958-63- fue elegido como el punto de partida de ese "gran salto adelante". Se adoptaron politicas económicas drásticas en línea con los principios maoístas y una política exterior agresiva como ingredientes de este paso radical. Pero las desastrosas consecuencias se sintieron ya en 1959: La Unión Soviética, que Mao declaró como revisionista, retiró el apoyo económico y técnico; crece la enemistad desarrollada con India y EE. UU. y la producción agrícola e industrial cae drásticamente. Estos combinados con los desastres naturales causaron la muerte de casi 20 millones de personas durante 1959-60.

Para 1961, la política maoísta fue abandonada y moderados como Liu Shaoqi, Deng Xiaoping y Chou Enlai, revirtieron el enfoque maoísta. Mao fue considerado responsable del desastre y renunció al cargo de jefe del estado -aunque mantuvo el cargo de presidente del partido-. Los tres moderados habian restringido el poder de Mao, pero para el pueblo chino común todavía era visto como el líder de la revolución y el Padre de la Nación. Quería usar su popularidad para resucitar su autoridad a expensas de los moderados. Entonces planeó lanzar la llamada Revolución Cultural ${ }^{2}$.

\section{La revolución cultural}

El objetivo básico de la Revolución Cultural, declaró Mao, sería la eliminación de los seguidores del camino capitalista -los liberales- del

${ }^{2}$ Ver http://www.historylearningsite.co.uk/great_leap_forward.htm 
poder en el partido comunista y restaurar el camino marxista de la lucha de clases. Los siguientes extractos de las directivas de Mao son evidencia de este enfoque de la Revolución Cultural.

1. "Los viejos socialdemócratas en las últimas décadas, y los revisionistas modernos en los últimos doce años más o menos, han formado un grupo de elementos anticomunistas, antipopulares $y$ contrarrevolucionarios contra quienes estamos librando una lucha a muerte. No hay igualdad entre nosotros y ellos. Por lo tanto, la lucha contra ellos es una lucha por nuestra preservación y su extinción. La relación entre nosotros y ellos nunca puede ser de igualdad; Es una relación de una clase que oprime a otra, es decir, una dictadura proletaria sobre la burguesía. El día en que la gente sea feliz será el dia en que los contrarrevolucionarios comiencen su miseria." (Mao, 2004, dt. 10-6-68)

2. "La contradicción básica que la gran Revolución Cultural proletaria está tratando de resolver es la que existe entre el proletariado y la burguesía, entre los caminos proletario y burgués. El punto principal del movimiento es luchar contra los seguidores del camino capitalistas con autoridad en el partido." (Ibíd. Dt. 24-9-1966)

3. "El problema básico de una revolución es el problema del poder político. La posesión del poder politico significa la posesión de todo, la pérdida del mismo significa la pérdida de todo." (Ibíd. Dt. 13-8-67)

Para luchar contra los oponentes, Mao quería tomar la ayuda de los estudiantes y las masas que podrian ser fácilmente conmovidos por la predicación emocional e idealista. Los Guardias Rojos formados principalmente por jóvenes estudiantes fueron dirigidos a atacar a los oponentes de Mao. 
1. "Los revolucionarios guardias rojos y las organizaciones revolucionarias estudiantiles deben formar una gran alianza. Mientras sean organizaciones de masas revolucionarias, deben formar una gran alianza de acuerdo con los principios revolucionarios."(Ibid. Dt. 1-10-67)

Mao también se dio cuenta de que sin control sobre la fuerza militar no sería posible combatir y acorralar a los oponentes.

Para ganar el apoyo de la fuerza militar, especialmente del Ejército Popular de Liberación -PLA- forjó una alianza con Lin Biao, el Ministro de Defensa, con la seguridad de que sería el sucesor de Mao. Mao presentó las siguientes pautas para Lin Biao y el Ejército en una carta a Lin Biao:

"Debes enviar tropas para apoyar a las amplias masas revolucionarias de izquierda. Más adelante, cuando los verdaderos revolucionarios necesiten el apoyo del PLA, usted debe hacer lo mismo. La llamada 'no interferencia' es falsa. El PLA ha estado involucrado por algún tiempo. Creo que sobre este asunto deberías emitir nuevos pedidos; los viejos deberian ser cancelados."(Ibid. dt. 23-1-67)

Ahora, en lo que respecta a la primera y más importante tarea de la alianza pro-Revolución Cultural estudiante-masa-ejército, Mao emitió la directiva:

“Bombardear el cuartel general". (Ibid. Dt. 5-8-66)

Entonces queda claro que, en contraste con la creencia común, la Revolución Cultural de Mao no tuvo nada que ver con el desarrollo moralista de la sociedad. Fue simplemente un esfuerzo por restaurar y fortalecer el camino erróneo de la lucha de clases, que finalmente degeneró en una lucha por el poder dentro del Partido Comunista de China. 


\section{Consecuencias}

Las consecuencias de la Revolución Cultural fueron simplemente devastadoras: el caos, la anarquía, la guerra civil, los incendios provocados, el saqueo, el daño sin sentido de la propiedad y los recursos productivos, los asesinatos, en resumen, la Revolución Cultural dejó a China en una condición experimentada por una tierra bajo un horrible cataclismo.

En el curso de los incidentes turbulentos, hubo un reordenamiento y reorganización del poder de un líder sobre otro. Al principio, resultó ser una lucha de poder entre dos grupos radicales liderados respectivamente por Lin Biao y Jiang Qing. Con la supremacía de este último grupo, Chiang fue arrestada y desapareció y Lin murió en un accidente aéreo mientras huía de China en 1971.

Pero luego el viento giró, ahora contra los radicales restantes liderados por Jiang Qing. Mao murió y luego Jiang Qing, la última partidaria de la Revolución Cultural, fue arrestada en 1976. La Revolución Cultural llegó a su fin dejando a China en ruinas. (Barnouin y Changgen, 1993), (Chen, 1975), (Domes, 1973), (Joseph et al., 1991), (Lee, 1978), (Tang, 1986).

\section{Adam Smith y el dilema humano}

De acuerdo con la obra maestra filosófica de Smith, "La teoría de los sentimientos morales", los sentimientos básicos del hombre se pueden dividir en dos categorias opuestas: interés propio y sentimientos similares. Smith supone que todos los sentimientos principales que pertenecen a cualquiera de estas dos categorias están dotados al hombre por la naturaleza.

"La gran división de nuestros afectos está en lo egoísta y lo benevolente" (Smith 1759, VII.II.4). 
Toda la ética humana pertenece a la segunda categoría de los sentimientos smithianos, a saber. "empatía". El progreso material se produce principalmente por el motivo de mejorar la propia condición, un motivo perteneciente a la categoria de "interés propio", y en su plena manifestación se convierte en el sentimiento dominante.

"Es esto lo que primero los impulsó a cultivar el suelo, construir casas, fundar ciudades y comunidades, e inventar y mejorar todas las ciencias y artes que ennoblecen y embellecen la vida humana, que han cambiado por completo la faz del mundo. Han convertido los rudos bosques de la naturaleza en agradables y fértiles llanuras, e hicieron del océano sin huellas y árido, un nuevo fondo de subsistencia y la gran vía de comunicación con las diferentes naciones de la tierra." (Ibid. IV.I.10) pero “... es la causa de todo el tumulto y el ajetreo, toda la rapiña e injusticia que la avaricia y la ambición han introducido en este mundo" (Ibid. I.III.23).

El sistema capitalista abre infinitas oportunidades para el avance material de los comerciantes e industriales, y en su impulso temerario hacia el logro de ganancias cada vez más altas, todos los sentimientos éticos, sentimientos y valores humanos están destinados a ser barridos.

La paradoja aquí es que el progreso materialista no es posible sin los sentimientos humanos más bajos de interés propio de la codicia y el deseo de poder, pero estos también son responsables de todas las enfermedades asociadas con el progreso materialista. La pregunta es cómo minimizar los impactos adversos del progreso materialista basado en el interés propio. Esto podría hacerse moderando el interés propio mediante la infusión de ética y sentimientos más nobles en la mente humana. Pero el obstáculo más serio en el camino de este objetivo puede llamarse el dilema de Smith. 


\section{El dilema smithiano}

En resumen, el Dilema Smithiano es que la psique de la mayoría de las personas admiran a los ricos y poderosos, incluso si estas personas han alcanzado su poder y riqueza a través de medios sin escrúpulos. Por otro lado, una persona honesta y virtuosa que es pobre y que carece de poder en la sociedad es descuidada, incluso despreciada por la gente común.

Profundizando en la psicología humana básica, Adam Smith opina que cualesquiera que sean sus propias posiciones, la mayoría de las personas alaban y adoran a las personas exitosas y ricas, independientemente de si sus éxitos se han logrado por medios éticos o poco éticos. Además, la mayoria de las personas aprecian en su subconsciente el deseo oculto de alcanzar la opulencia y la fama para obtener elogios y la aprobación de todos y cada uno.

"Esta disposición a admirar, y casi a adorar, a los ricos y poderosos, y a despreciar, o, al menos, descuidar a las personas de condición pobre y mala, aunque es necesario tanto para establecer como para mantener la distinción de los rangos y el orden de la sociedad, es, al mismo tiempo, la gran y más universal causa de la corrupción de nuestros sentimientos morales. Esa riqueza y grandeza a menudo se consideran con el respeto y la admiración que se deben solo a la sabiduria y la virtud; y que el desprecio, del cual el vicio y la insensatez son los únicos objetos apropiados, a menudo se otorga injustamente a la pobreza y la debilidad, ha sido la queja de los moralistas en todas las épocas." (Ibid. I.III.28)

"Deseamos que ambos sean respetables y respetados. Tememos que ambos sean despreciables y condenados. Pero, al venir al mundo, pronto descubrimos que la sabiduría y la virtud no son en absoluto los 
únicos objetos de respeto; ni vicio y locura, de desprecio. Frecuentemente vemos las atenciones respetuosas del mundo más fuertemente dirigidas hacia los ricos y los grandes que hacia los sabios y los virtuosos." (Ibid. I.III.29)

"En igual grado de mérito, escasea cualquier hombre que no respete más al rico y al grande que al pobre y al humilde. Con la mayoria de los hombres, la presunción y la vanidad de los primeros son mucho más admirados que el mérito real y sólido de los últimos." (Ibid. I.III.31)

\section{Solución Sankhya}

Según la filosofia de Sankhya, la conciencia humana es parte de la manifestación material de la naturaleza y es la combinación de tres modos, a saber: satva, rajas y tamas, dotados por la naturaleza. Todos estos modos básicos se combinan en diferentes grados para asignar diferentes características a diferentes individuos. Si se aísla en abstracto, la satva sin mezclar pertenece a la bondad y la virtud; rajas a la pasión y el deseo insaciable y tamas a la oscuridad de la mente, la obsesión y la inercia. Todas nuestras facultades mentales e intelectuales se originan en estos tres modos básicos (Ballantyne, 2004).

E1 creador es omnipresente pero Nirguna Brahman es sin atributos.

Los seres humanos son saguna, que tienen atributos especificos y que son de tres categorias, a saber. I tamasika, dominada por el modo 'tamas'; II rajasika, dominada por el modo 'rajas' y III satvika, dominado por el modo 'satva'. Las personas satvika se caracterizan por cualidades más nobles, como la abstinencia, el sacrificio personal, el amor, la filantropía, la misericordia, la confianza en sí mismo, la diligencia y la compostura, mientras que las personas rajasika y tamasika poseen 
varias combinaciones de cualidades más básicas, como la codicia, la envidia, el odio, la ira, egoísmo, lujuria, ociosidad, crueldad y orgullo.

Ahora tomemos otro concepto de filosofia hindú, a saber. El concepto de ripu. En general, ripu significa enemigo. Estos ripus en la filosofia hindú son excesos de algunos instintos básicos o reflejos de la mente o el intelecto humano. Estos instintos básicos son necesarios para nuestra existencia material. Entonces, como tales, no son enemigos. Pero para tener efectos beneficiosos deben ser equilibrados y mantenerse bajo nuestro control. Si están en exceso, se convierten en nuestros enemigos y producen efectos desastrosos tanto para nosotros como para la sociedad. Los eruditos occidentales a menudo traducen erróneamente ripu como pecado. Pero los ripus no son pecados, sino razones de pecados o vicios. Hay seis ripus, a saber. Kama -deseo de placer material, deseo sexual, lujuria-, krodha-ira-, lobha-codicia-, moha-infatuación, apego, obsesión-, mada-orgullo, vanidad, arrogancia-, matsarya-envidia-.

Los instintos básicos, pertenecientes al ripus, pueden usarse para bien o para mal y por sí mismos son neutrales. Nuestro esfuerzo no debe ser eliminarlos, sino controlarlos y convertirnos en sus amos. El deseo sexual es normal y bueno, pero obsesionarse con el sexo y usarlo para dañar no lo es. La ira también es un elemento necesario que nos hace humanos: sin ella no podemos tener respetabilidad. La avaricia es una forma de deseo: puede ser un deseo tonto insaciable o un deseo positivo que puede usarse como un incentivo para el progreso. Del mismo modo, la vanidad y el orgullo son necesarios para la autoestima y la envidia puede fomentar una competencia saludable. Si los instintos básicos se mantienen bajo control, siempre son beneficiosos, pero si nos superan, se convierten en enemigos y nos arruinan a corto o largo plazo.

Bajo el modo satva, los ripus están completamente controlados y resultan benignos: residen en esa persona en perfecta armonía con la 
vida ética. Por otro lado, bajo los modos rajas y tamas, los ripus se vuelven dominantes y toman el control de las personas involucradas y, por lo tanto, se vuelven desastrosos tanto para los individuos involucrados como para la sociedad en general.

Según el análisis anterior, el avance de la sociedad humana y la eliminación de enfermedades exigen la transición de los estados tamas y rajas al estado satva. Sin embargo, ningún texto hindú antiguo proporciona pautas específicas para iniciar este proceso de transición. Solo el Kulanava Tantra - un texto del siglo VII- propone el camino de Kaula como un camino de autorrealización y un conjunto de técnicas para que los hombres, como un todo y sin distinción de casta, crezcan moralmente para alcanzar la misma dignidad humana, con independencia de sus circunstancias de nacimiento. Este texto propone enseñar a leer y escribir incluso a los parias, y una vez dentro del clan, un paria que había alcanzado un nivel de educación superior y tenía suficientes méritos, estaba por encima de un Brahmana recién iniciado. Dentro del clan, gobernó la meritocracia (Gómez, 2017). Los seguidores del clan Kaula y los practicantes de Vajrayana -budismo tántrico-, fueron exterminados en la India al final del imperio Sailendras, que los patrocinó y protegió entre los siglos VIII y XIII. Sus textos y sus enseñanzas liberadoras, destruidas. Algunas de estas enseñanzas se conservaron en Asia Central y China (Acri, 2017), quedando el budismo temprano y el tantrismo en la India totalmente corrompidos y demonizados por el sistema bramanico ${ }^{3}$.

Mao seguramente se ha referido a esta fuente cuando se definió como un adepto tántrico (Chhaya, 2007) y le preguntó al Dalai Lama por qué,

\footnotetext{
${ }^{3}$ Solo durante un corto período, las enseñanzas de Buda, Kabir y Nanak fueron más allá de la comunidad, la religión, el idioma, la cultura y la nación para proporcionar una panacea universal y un paradigma de igualdad, cuestionando las discriminaciones basadas en el nacimiento y el género y dando la bienvenida a hombres y mujeres a la Sangha. El hinduismo floreció solo después de la destrucción masiva del budismo temprano y del tantrismo (Bose, 2019).
} 
siendo él mismo un practicante tántrico, no había encabezado un movimiento para liberar a los campesinos de la opresión y la ignorancia (Gómez, 2013). De aquí viene la relevancia del concepto de la Revolución Cultural.

\section{Conclusión}

\section{De la Revolución Cultural a la evolución cultural}

Si analizamos con una visión profunda, las causas de las enfermedades de la sociedad humana tanto de Sankhya como de Smith se reducirian en esencia a lo mismo: las causas de las enfermedades en la sociedad humana se encuentran profundamente en los vicios básicos de la mente humana, a saber: la avaricia, el orgullo, los celos, el odio y la salida radica en infundir en la sociedad humana en su conjunto valores humanos más nobles como el amor, el sacrificio, la empatía, el sentimiento de compañerismo, la filantropía $\mathrm{y}$, al hacerlo, minimizar gradualmente los impactos adversos de los vicios básicos. No es realista pensar que todos los vicios básicos podrian erradicarse o eliminarse por completo. Sin embargo, se podría lograr un equilibrio entre las fuerzas más bajas y las fuerzas más nobles.

Ahora surge la pregunta sobre los procesos sociales que probablemente realicen esta tarea altamente deseable pero intrincada. $\mathrm{Ni}$ Sankhya ni Adam Smith proporcionan ningún medio viable y efectivo para este fin.

Aquí viene la relevancia de la Revolución Cultural, que en esencia, significa un proceso, que a través de los esfuerzos sociales colectivos, es probable que infunda valores más nobles y humanitarios en la sociedad en su conjunto. El problema con los maoístas, y de hecho el enfoque marxista básico de la Revolución Cultural, era que mezclaba este proceso 
social con la lucha de clases y los medios violentos. Esta es la razón básica del fracaso de la Revolución Cultural en China y la consiguiente destrucción de la propiedad, la pérdida de vidas y, sobre todo, el caos y la confusión que llevan a la economía china, la política y la sociedad en su conjunto a la depresión.

Sin embargo, podemos disociar el concepto de Revolución Cultural de las fantasias marxistas y maoístas de la lucha de clases y los medios violentos destructivos, y llegar a la connotación general del concepto, a saber. Un proceso social de purificación de las fuerzas psíquicas negativas que conducen a enfermedades de la sociedad. Sin embargo, el término "revolución" puede significar un medio violento de cambio radical en un corto período de tiempo. Por lo tanto, podemos usar mejor el término "Evolución Cultural" para evitar confusiones. Por lo tanto, la evolución cultural puede concebirse como un proceso evolutivo a largo plazo de modificación de los valores humanos y de la actitud mental.

Las siguientes tres preguntas surgen en relación con este concepto de Evolución Cultural:

¿Con qué manifestaciones específicas de vicios básicos se debe comenzar?, ¿Cómo lograr esto? y ¿Qué agencias desempeñarán un papel crucial a este respecto.

Vamos a elaborar la pregunta básica como se mencionó anteriormente.

Q1. ¿A qué manifestaciones específicas de vicios básicos deben ser dirigidas?

Las principales manifestaciones de vicios básicos a los que debe dirigirse para comenzar son:

1. Supersticiones religiosas y otras.

2. Ritos religiosos innecesarios y gravosos. 
3. Devoción ciega a los sacerdotes religiosos y gurúes.

4. Devoción ciega a partidos políticos y líderes políticos.

5. Devoción ciega a todas las categorías de celebridades.

6. División de la sociedad sobre la base de la casta, el credo, la lengua materna y la religión y las reservas, la intocabilidad, la discriminación en base a ellos.

7. Actitud de Tamasika en actividades de partidos politicos y medios de comunicación

Q2: ¿Cómo realizar las tareas anteriores?

Las medidas inmediatas a emprender para iniciar el proceso de la Evolución Cultural son las siguientes:

A. Rápida industrialización y avance tecnológico -modos de producción rajasika- en todas las esferas de la economia para erradicar completamente todos los vestigios de los modos de producción precapitalistas -tamasika-.

B. Eliminación completa del analfabetismo.

C. Difusión de la educación moral y científica para inculcar la perspectiva científica y ética del mundo.

Q3: ¿Cuáles deberian ser las principales agencias participativas

Intelectuales iluminados, ONG's, gobiernos, medios de comunicación, instituciones educativas.

Es probable que el obstáculo más obstinado en el camino de iniciar la evolución cultural sea la psique humana básica discutida en la sección III como el dilema de Smith.

Entonces, una tarea importante al comienzo de la iniciación de la Evolución Cultural sería enfrentar el serio obstáculo relacionado con el 
dilema Smithiano. Este es uno de los obstáculos más serios y se deben idear métodos para abordarlo. Considerando la gravedad de este problema, es necesario discutir el tema en detalle en otro artículo más completo.

Créditos: Traducción del original en inglés publicado en la Revista Científica Arbitrada de la Fundación MenteClara (Basu, 2018) 


\section{Referencias}

Acri, A. (2017). "Viajando por los "Caminos del sur": El budismo esotérico en el Asia marítima, siglos VII-XIII d. C.». Revista Cientifica Arbitrada de la Fundación MenteClara, 2(2), 6-62. DOI: $10.32351 /$ rca.v2.2.28 http://fundacionmenteclara.org.ar/revista/index.php/RCA/article/view/28

Basu R. L. (2018). "From Cultural Revolution to Cultural Evolution». Revista Científica Arbitrada de la Fundación MenteClara, 3(1), 7-28. DOI: 10.32351/rca.v3.1.37 https://fundacionmenteclara.org.ar/revista/index.php/RCA/article/view/37

Ballantyne, James R. (translator) (2004), Sankhya Aphorisms of Kapila (Sanskrit Text with English Translation), Revised New Edition, Delhi, India Parimal Publications.

Barnouin, Barbara and Yu Changgen (1993), Ten Years of Turbulence, London, Kegan Paul International.

Bose A. L. (2019). "Towards a Comparative poetics of Buddha, Kabir and Guru Nanak: From A Secular Democratic perspective». Revista Científica Arbitrada de la Fundación MenteClara, 4(2) abril-septiembre 2019, 19-44. DOI: 10.32351/rca.v4.2.85 https://fundacionmenteclara.org.ar/revista/index.php/RCA/article/view/85

Chen, Jac (1975), Inside the Cultural Revolution, New York, MacMillan.

Chhaya, M. (2007) Dalai Lama. Man, Monk, Mystic, Doubleday, 2007

Domes, Jurgen (1973), The Internal Politics of China 1949-72, New York, Praeger.

Gómez, O. R. (2013). El tantrismo dentro de la Compañía de Jesús - del Tíbet al Vaticano hoy. Buenos Aires, Argentina: Ediciones Menteclara. http://tantra.org.ar/biblioteca/index.php/Biblioteca/article/view/4/5

Gómez, O. R. (2017). "Análisis crítico del Kularnava Tantra -Parte 1- Un prólogo viciado de sexualidad reprimida». Revista Científica Arbitrada de la Fundación MenteClara, 2(1), 114-141. DOI: $10.32351 /$ rca.v2.1.26

http://fundacionmenteclara.org.ar/revista/index.php/RCA/article/view/26

Joseph, William et al. (eds.) (1991), New Perspectives on the Cultural Revolution, Cambridge, MA, Harvard University Press.

Lee, Hongyong (1978), The Politics of the Chinese Cultural Revolution, Berkeley, CA, University of California Press. 
Mao Tse-tung (2004), "Directives Regarding Cultural Revolution 1966-69”, Selected Works Vol. IX, Secunderabad, Kranti publications. https://www.marxists.org/reference/archive/mao/selected-works/volume9/mswv9 84.htm

Smith, Adam (1759), The Theory of Moral Sentiments. London, A. Millar, Sixth edition, 1790.

Tang, Tsou (1986), The Cultural Revolution and post-Mao reforms: a historical perspective, Chicago. University of Chicago Press.

Telang, Kashinath Trimbak (translator) (1882), The Bhagavad Gita, The Sacred Books of the East, Vol.8, Oxford Clarendon Press.

Vijnanananda, Swami (translator) (1921-22), Srîmad Devi Bhagavatam (Devi Purana), (Book-7, Chapter-35, Slokas-2-3), Scanned, and proofed by Sahaji, 2004-5.

http://upload.vedpuran.net/Uploads/68655SrimadDeviPurana.pdf 